\title{
Tracking Maneuvering Trajectory under a Boosted Kalman Filtering
}

\author{
Le Zhang ${ }^{1,2, *}$, Zhiyong $\mathrm{An}^{1}$, Yongping $\mathrm{Hao}^{2}$ and Qin Dai ${ }^{2}$ \\ ${ }^{1}$ Opto-Electronic Engineering Department, Changchun University of Science \\ and Technology, Changchun, China \\ ${ }^{2}$ Equipment Engineering Department, Shenyang Ligong University, Shenyang, \\ China \\ zhangle_sylu@126.com
}

\begin{abstract}
In this paper, an adaptive Kalman Filtering method is presented for the state prediction of random systems. It is shown that the adaptive Kalman Filtering method in conjunction with equilibrium optimization solution can estimate the intial accelerations of targets effectively since the equilibrium optimization solution tunes the state prediction vector to diminish the error between measured value and prediction estimation value. We evaluate our model on special and random trajectories. Experimental evidence shows that the proposed method can robustly estimate an initial acceleration from a dynamic model and stably track a trajectory which is moving randomly.
\end{abstract}

Keywords: Kalman Filtering, manewering trajectory initial velocity

\section{Introduction}

Target trajectory tracking is fundamentally a problem of estimating current and future target motion parameters Filtering and prediction are necessary technical means to obtain them, such as position, velocity and acceleration, etc. Accurate tracking is crucial for many applications, such as space-based early warning, radar trajectory prediction system, inertial navigation system [1-3]. Despite enormous progress in recent years, the tracking abilities of humans still easity outperform state-of-the-art algorithms in real world, both in terms of preciston and accuracy, if target is moving in a non-maneuvering state [3]. During target estimation and prediction in fact, when target tends to maneuver, the problem becomes much more complicated. Unfortunately, in the realm of trajectory tracking typical filtering, prediction measure and motion pre-model do not lead to meet the need of the problem solving. The estimation and prediction are so often divergent that have to be taken into account.

In ths york we investigate the question whether it is really beneficial for trajectory tracking to improve Kalman Filtering model to solute the problem effectively. We attempt to design a new adaptive method such that it offers equilibrium optimization solution to diminish the error between measured value and prediction estimation value. For the purpose of this paper, the standard Kalman Filtering can be applied to track a nonmaneuvering state trajectory [4,5]. Then by choosing the new adaptive Kalman Filtering as the filtering algorithm, it is shown that the adaptive algorithm yields more accurate results than the standard Kalman Filtering for the non-maneuvering and maneuvering target trajectories. 


\section{Tracking Algorithm}

\subsection{Linear Kalman Filtering for Discrete-Time Systems}

\subsubsection{White Noise and Color Noise}

The random process $w(t)$ in (1) and (2):

$$
\begin{gathered}
\mathrm{E}[w(t)]=0 \\
\mathrm{E}\left[w(t) w(\tau)^{\mathrm{T}}\right]=\rho_{w} \delta(t-\tau)
\end{gathered}
$$

where $w(t)$ is white noise process, in this model, $\rho_{w}$ is the covariance of $w(t), \delta(t-\tau)$ is the Dirac- $\delta$ function

$$
\delta(t-\tau)=\left\{\begin{array}{ll}
0 & t=\tau \\
\infty & t \neq \tau
\end{array}, \quad \int_{-\infty}^{\infty} \delta(t-\tau) d \tau=1\right.
$$

Equation (2) is the autocorrelation function of $w(t)$, can be expressed as

$$
R_{w}(t-\tau)=\rho_{w} \delta(t-\tau)
$$

It shows that the autocorrelation function is related to interval $(t-\tau)$ but with no relationship with time $t$, so $w(t)$ is a smooth process.

Let $\mu=t-\tau$, the power spectrum of $w(t)$ is as follows

$$
s_{w}(\omega)=\int_{-\infty}^{\infty} \rho_{w} \delta(\mu) e^{-\mathrm{j} \omega \omega} d \mu=\mathcal{R}_{w}
$$

If $\{w(k)\}$ is a random sequence and satisfies

$$
\mathrm{E}\left(w_{k}\right)=0
$$

$$
1 \mathrm{E}\left(w_{k} w_{i}^{\mathrm{T}}\right)=Q_{k} \delta_{k j}
$$

then $w(k)$ is a white noise sequence, $Q_{k}$ and $\delta_{k j}$ are covariance matrix and Kronecker- $\delta$ function, shown as follows, of $w(k)$, Tespectively [6].

\subsubsection{Random Linear Discrete System Model}

Given that state and observation equations of random linear discrete system are

$$
\begin{gathered}
x_{k}=\Phi_{k \mid k-1} x_{k-1}+\Gamma_{k \mid k-1} w_{k-1} \\
z_{k}=H_{k} x_{k}+v_{k}
\end{gathered}
$$

where $x_{k} \in R^{n}$ is the system state vector, $z_{k} \in R^{m}$ is the measurement vectors, $\Phi_{k \mid k} \in R^{n \times n}$ is the state transition matrix, $\Gamma_{k \mid k-1} \in R^{n \times p}$ is the noise input matrix, $H_{\mathcal{K}} \in R^{m \times n}$ is the measurement matrix, $w_{k-1} \in R^{p}$ is the system process noise, $v_{k} \in R^{m}$ is the measurement noise.

The statistical characteristics of the system process noise and measurement noise are assumed as

$$
\left\{\begin{array}{cc}
\mathrm{E}\left(w_{k}\right)=0, & \operatorname{Cov}\left(w_{k}, w_{j}\right)=Q_{k} \delta_{k j} \\
\mathrm{E}\left(\mathrm{v}_{k}\right)=0, & \operatorname{Cov}\left(\mathrm{v}_{k}, \mathrm{v}_{j}\right)=R_{k} \delta_{k j} \\
\operatorname{Cov}\left(\mathrm{w}_{k}, \mathrm{v}_{j}\right)=0 &
\end{array}\right.
$$


where $Q_{k}$ is the non-negative definite covariance matrix, $R_{k}$ is the positive definite covariance matrix, $S_{k}$ is the cross covariance matrix.

If $z_{k}$ satisfies (10) constraints, then state estimation $\hat{x}_{k}$ is in accordance with the following equations:

Step state prediction

$$
\hat{x}_{k \mid k-1}=\Phi_{k \mid k-1} \hat{x}_{k-1}
$$

State estimation

$$
\hat{x}_{k}=\hat{x}_{k \mid k-1}+K_{k}\left(z_{k}-H_{k} \hat{x}_{k \mid k-1}\right)
$$

Filtering gain matrix

$$
K_{k}=P_{k \mid k-1} H_{k}^{T}\left(H_{k} P_{k \mid k-1} H_{k}^{T}+R_{k}\right)^{-1}
$$

Step prediction error covariance matrix

Estimation error covariance matrix

$$
P_{k \mid k-1}=\Phi_{k \mid k-1} P_{k-1} \Phi_{k \mid k-1}^{T}+\Gamma_{k \mid k-1} Q_{k-1} \Gamma_{k \mid k-1}^{T}
$$

$$
P_{k}=\left(I-K_{k} H_{k}\right) P_{k \mid k-1}
$$

Equation (12) 16) are basic equations of kalman filtering for random /near discrete systems. If the initial values $\hat{x}_{0}$ and $P_{0}$ are given state estimation $\hat{x}_{k}$ at time $k$ can be recursive according to the measured values $z_{k}$.

\subsection{Target Tracking Algorithm}

As was mentioned above, when the Carget motion mathematical model is precise, such as uniform linear motion or uniform circtiar motion the results of prediction and filtering are satisfactory by applying the standard Kalman-Filtering. However, when the target motion model is not known, it is unable to establish accurate mathematical models for the target. At time $k$, generally we can assime that at any moment, targets are in uniform linear motion, thereby establish mathematical models. But this will lead to degradation on tracking accuracy, in some cases, evên cause filtering divergence.

In this section, the target trajectory is random, at time $k$, target motion parameters $x_{k}$ are random To overcome this problem, based on the analysis of motion characteristics of maneuvering targets, the modification to state prediction vector $\hat{x}_{k \mid k-1}$ at time $k$ is introduced to improye the prediction on the real value, so as to get a better performance than the standard Kalon Filtering [7-10].

Target maneurering model

$$
\begin{gathered}
x_{k}=\Phi_{k \mid k-1} x_{k-1}+\Gamma_{k \mid k-1} w_{k-1} \\
z_{k}=H_{k} x_{k}+v_{k}
\end{gathered}
$$

where $\Phi_{k \mid k-1}$ is unknown. At time $k$, it is assumed that the real kinematic parameters of the target are

Observation vector is

$$
X_{k}=\left[r x_{k}, r y_{k}, v x_{k}, v y_{k}\right]^{T}
$$

When $k>3$, we have

At time $k-1$, observation vector is

$$
z_{k}=\left[z x_{k}, z y_{k}\right]^{T}
$$

$$
z_{k-1}=\left[z x_{k-1}, z y_{k-1}\right]^{T}
$$

At time $k-2$, observation vector is 
At time $k-3$, observation vector is

$$
z_{k-2}=\left[z x_{k-2}, z y_{k-2}\right]^{T}
$$

$$
z_{k-3}=\left[z x_{k-3}, z y_{k-3}\right]^{T}
$$

At time $k-1$, average velocity is defined as follows:

$$
v_{k-1}=\left[v x_{k-1}, v y_{k-1}\right]^{T}=\left(\left[z x_{k-1}, z y_{k-1}\right]^{T}-\left[z x_{k-2}, z y_{k-2}\right]^{T}\right) / T
$$

At time $k-2$

$$
v_{k-2}=\left[v x_{k-2}, v y_{k-2}\right]^{T}=\left(\left[z x_{k-2}, z y_{k-2}\right]^{T}-\left[z x_{k-3}, z y_{k-3}\right]^{T}\right) / T
$$

At time $k-1$, average acceleration is

$$
a_{k-1}=\left[a x_{k-1}, a y_{k-1}\right]^{T}=\left(v_{k-1}-v_{k-2}\right) / T
$$

State prediction vector at time $k$ after modified is

where

$$
\hat{x}_{k \mid k-1}=F_{k \mid k-1} \hat{x}_{k-1}+\left[0,0, v x_{k-1}, v y_{k-1}\right]^{T}+\Gamma_{k \mid k-1} a_{k-1}
$$

$$
F_{k \mid k-1}=\left[\begin{array}{cccc}
1 & 0 & T & 0 \\
0 & 1 & 0 & T \\
0 & 0 & 0 & 0 \\
0 & 0 & 0 & 0
\end{array}\right], \quad \Gamma_{k \mid k-1}=\left[\begin{array}{cc}
T^{2} / 2 & 0 \\
0 & T^{2} / 2 \\
T & 0 \\
0 & T
\end{array}\right]
$$

Prediction error covariance matrix is

$$
P_{k \mid k-1}=\Phi_{k \mid k-1} P_{k+1} \Phi_{k \mid k-} T+\Gamma_{k \mid k-1} Q_{k-1} P_{k \mid k-1}^{T}
$$

where

$$
\Phi_{k \mid k-1}=\left[\begin{array}{cccc}
1 & 0 & T & 0 \\
0 & 1 & 0 & T \\
0 & 0 & 1 & 0 \\
0 & 0 & 0 & 1
\end{array}\right], Q_{k-1}=\left[\begin{array}{cc}
10 & 0 \\
0 & 10
\end{array}\right]
$$

Filtering gain mâtrix is

$$
K_{\mathrm{R}}=P_{k \mid k-1} H_{k}^{T}\left(H_{k} P_{k \mid k-1} H_{k}^{T}+R_{k}\right)^{-1}
$$

where

$$
H_{k}=\left[\begin{array}{llll}
1 & 0 & 0 & 0 \\
0 & 1 & 0 & 0
\end{array}\right], \quad R_{k}=\left[\begin{array}{cc}
5000 & 0 \\
0 & 5000
\end{array}\right]
$$

State estimation is

$$
\hat{x}_{k}=\hat{x}_{k \mid k-1}+K_{k}\left(z_{k}-H_{k} \hat{x}_{k \mid k-1}\right)
$$

Estimation error covariance matrix is

$$
P_{k}=\left(I-K_{k} H_{k}\right) P_{k \mid k-1}
$$

Filtering initialization:

$\hat{x}_{1}=[123,234,100,200]^{T}, \hat{x}_{2}=[234,345,100,200]^{T}, \hat{x}_{3}=[345,456,100,200]^{T}$

Filtering error covariance matrix is

$$
P_{0}=\left[\begin{array}{llll}
1 & 0 & 0 & 0 \\
0 & 2 & 0 & 0 \\
0 & 0 & 1 & 0 \\
0 & 0 & 0 & 2
\end{array}\right]
$$




\section{Experiments Test}

\subsection{Non-maneuvering Trajectory Tracking Simulations}

The non-maneuvering trajectory is a semi circular curve; the target is still in a state of uniform motion. The simulation results are given by Figure 1, Figure 2 and Figure 3. It is shown that the standard Kalman Filtering provides good accuracy in estimating the target trajectory which can be modeled accurately.

Although the trajectory is in the state of uniform circular motion, it can get accurate mathematical model. Figure 1, Figure 2 and Figure 3 show that Kalman Filtering still converge and achieve the satisfied performance.

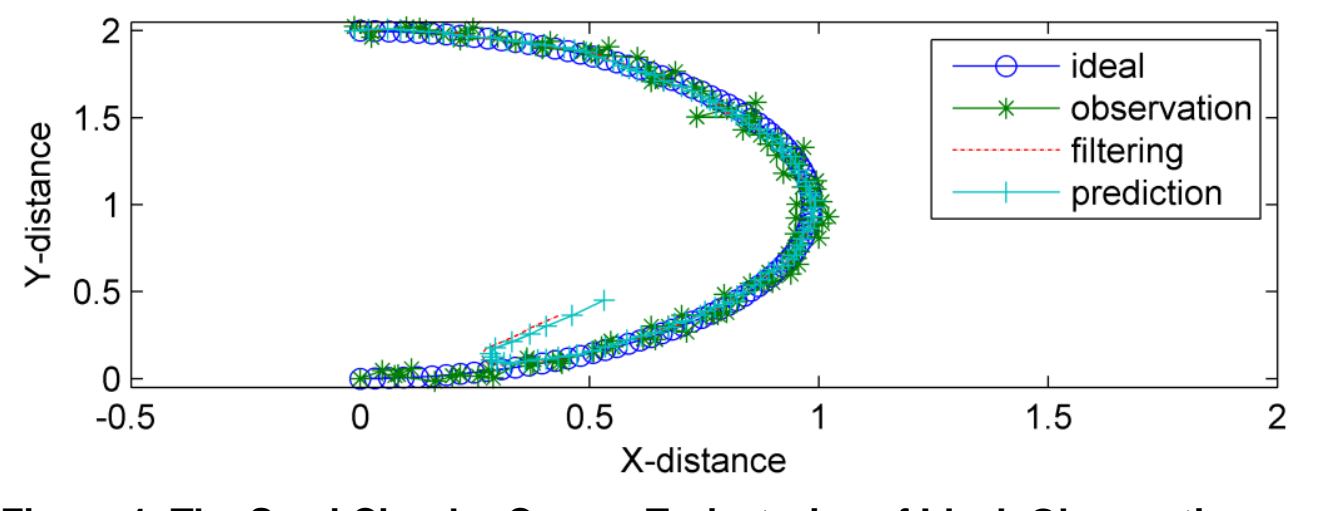

Figure 1. The Semi Circular Curves Trajectories of Ideal, Observation, Filtering and Prediction

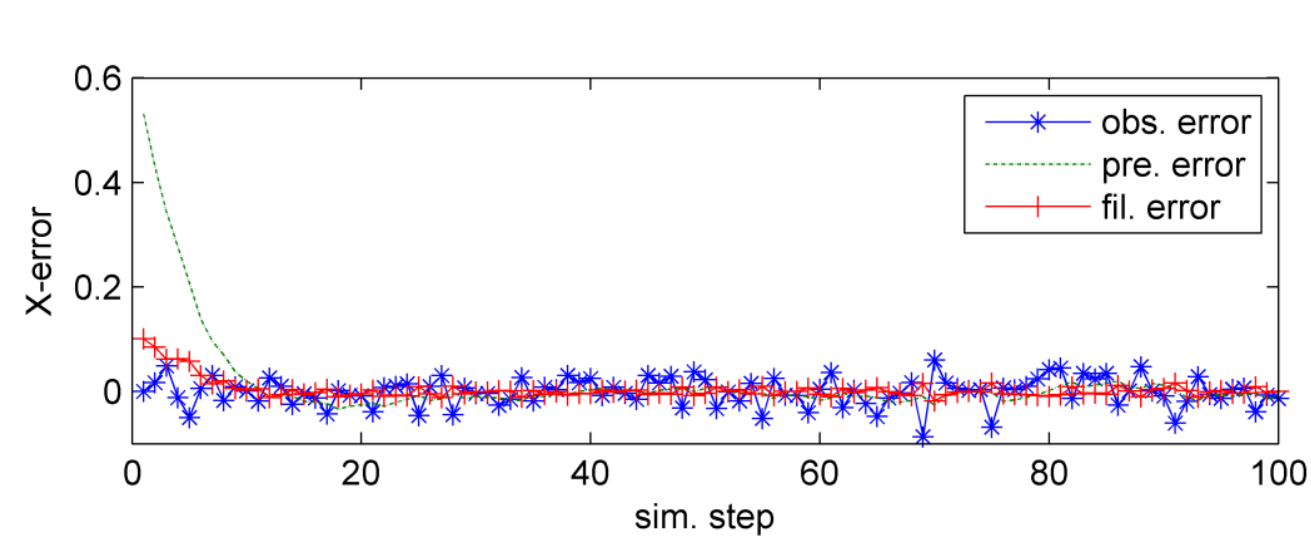

Figure 2. The Error of Observation, Prediction and Filtering In X

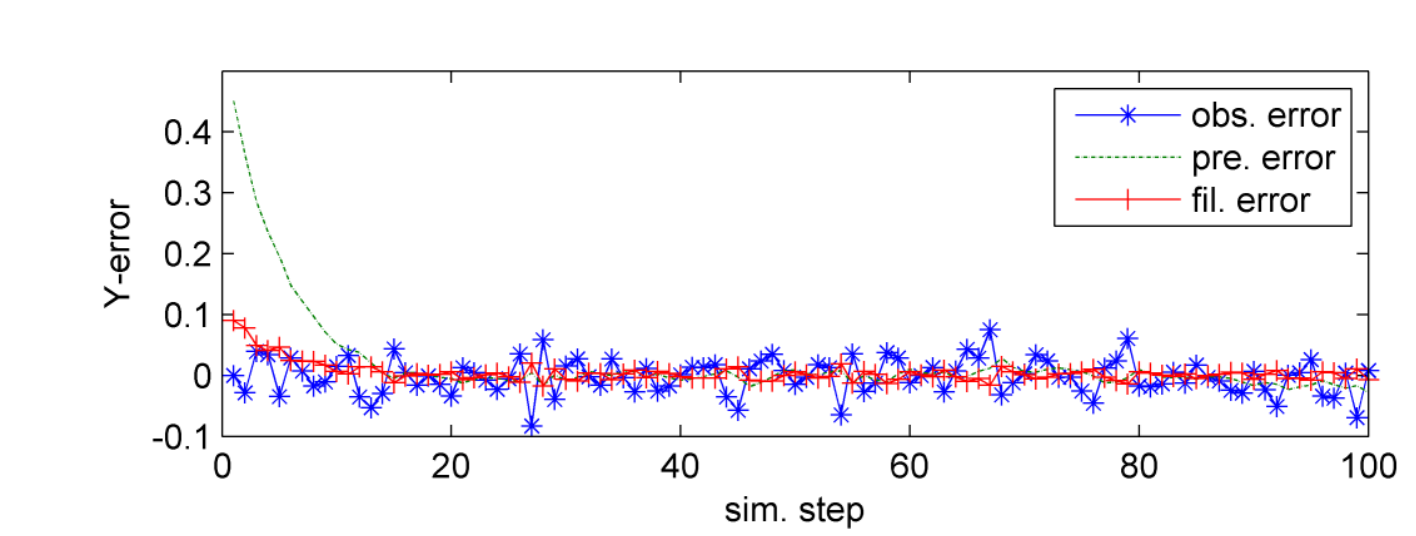

Figure 3. The Error of Observation, Prediction and Filtering In $Y$ 


\subsection{Random Trajectory Tracking Simulation}

In this simulation, the motion model can not be established, the traditional way is to assume that the target is still in uniform linear motion, but the actual motion parameters can not be predicted, it will inevitably lead to a decline in the tracking accuracy sometimes even lead to filter divergence.

In view of this situation, this paper constructs a new improved Kalman Filtering algorithm. As described above, by improving the prediction value to reduce the error. The simulation results given by Figure 4 9 show that the improved algorithm declines faster than standard methods but the tracking accuracy is greatly improved.

\subsubsection{Standard Kalman Filtering Algorithm}

Figure 5 and Figure 6 show filter errors in $\mathrm{X}$-axis and $\mathrm{Y}$-axis, we can see that the filter error in $\mathrm{X}$-axis is even larger than the observation error, in this case algorithm has lost its practical significance.

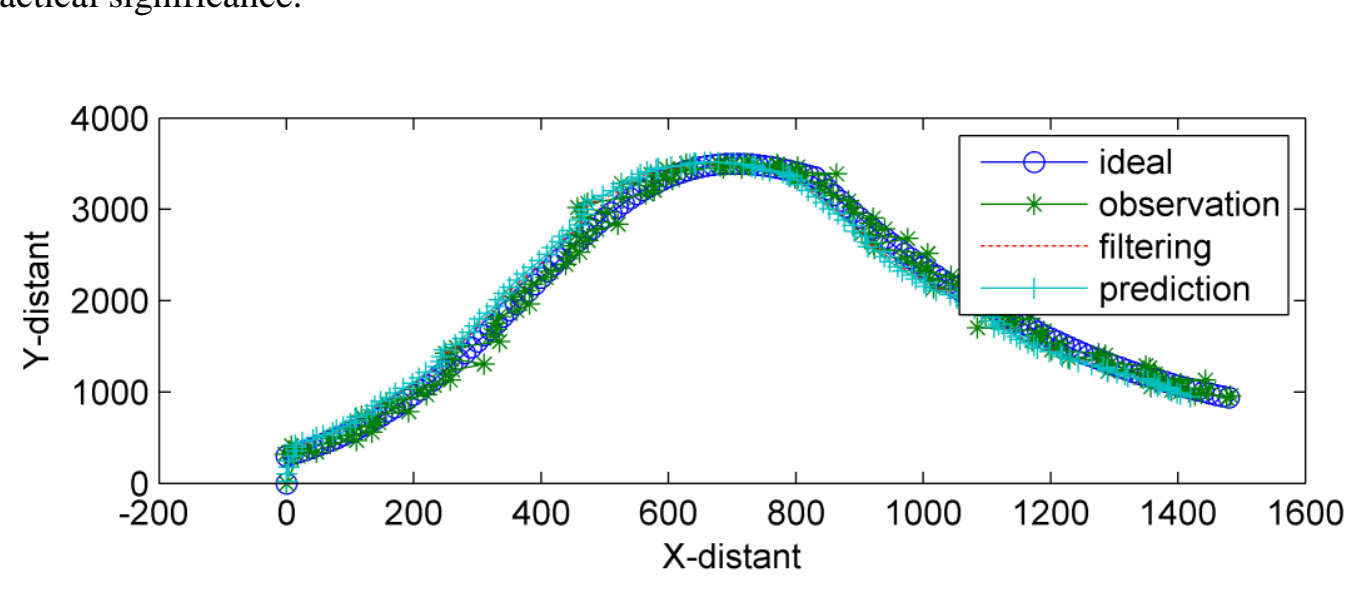

Figure 4. The Maneuvering Trajectories of Ideal, Observation, Filtering and

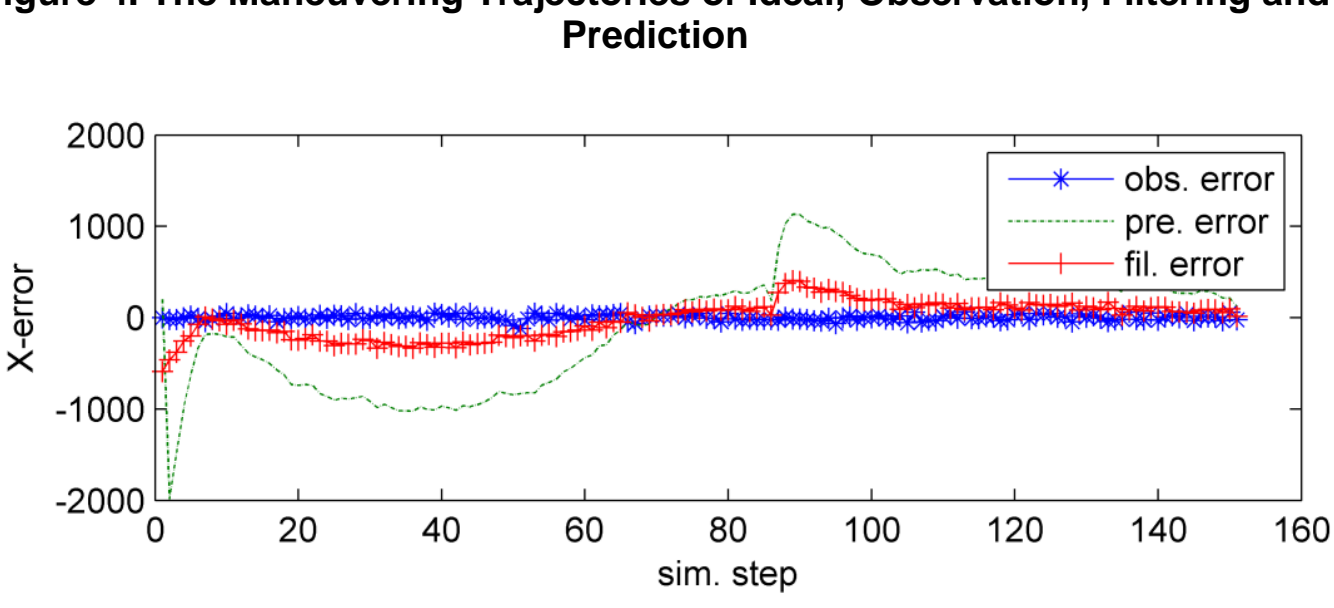

Figure 5. The Error of Observation, Prediction and Filtering $\ln X$ 


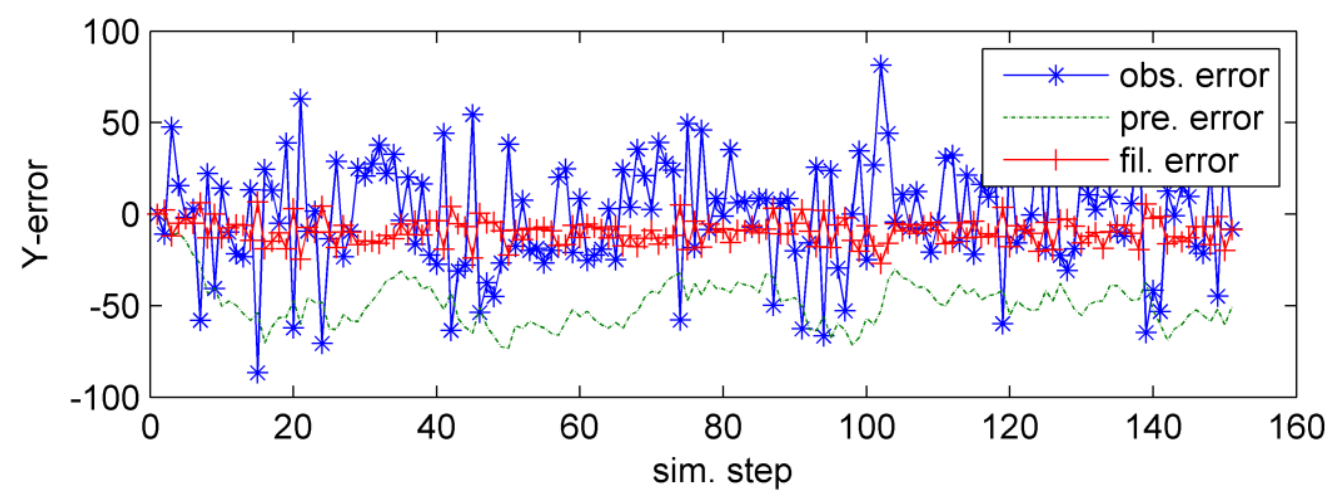

Figure 6. The Error of Observation, Prediction and Filtering In $Y$

\subsubsection{Improved Kalman Filtering Algorithm}

Figure 7, Figure 8 and Figure 9 demonstrate that the performance sûch as tracking accuracy, convergence rate and other indicators, of the improved algorithm on tracking the same maneuvering curve has risen with respect to that of the standard method. It is noted that the initial estimation value is very large however the improved algorithm for tracking a maneuvering motion can still rapidly converge.

The error curves are shown in Figure 8 and Figure 9 . The case that although the observed trajectory and the predict trajectory both present large errors, the filtering trajectory by applying the improved algorithm can stilget better filtering accuracy. From Figure 8, we can see that the local peak value of he prediction error in X-axis is 237.4755. Similarly from Figure 9 we can see that the local peak value of prediction error in Y-axis is 165.4967 . From the figures, during the entresimulation process, the observation errors fluctuate wildly, and the same result occures to the prediction error. This indicates that the tracking environment is quite bad, however the filtering error curve remains relatively small fluctuations, showing greater vitality of the improved algorithm, compared to the standard algorithms in dealing with the the tracking problem in harsh conditions.

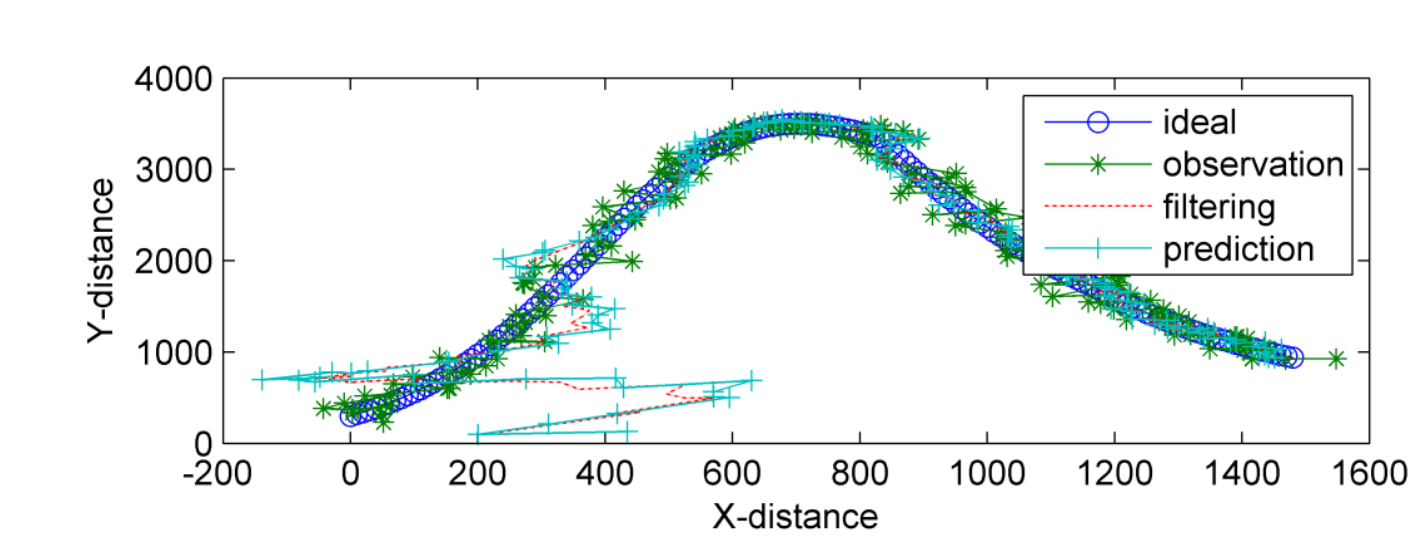

Figure 7. The Maneuvering Trajectories of Ideal, Observation, Filtering and Prediction in Adaptive Algorithm 


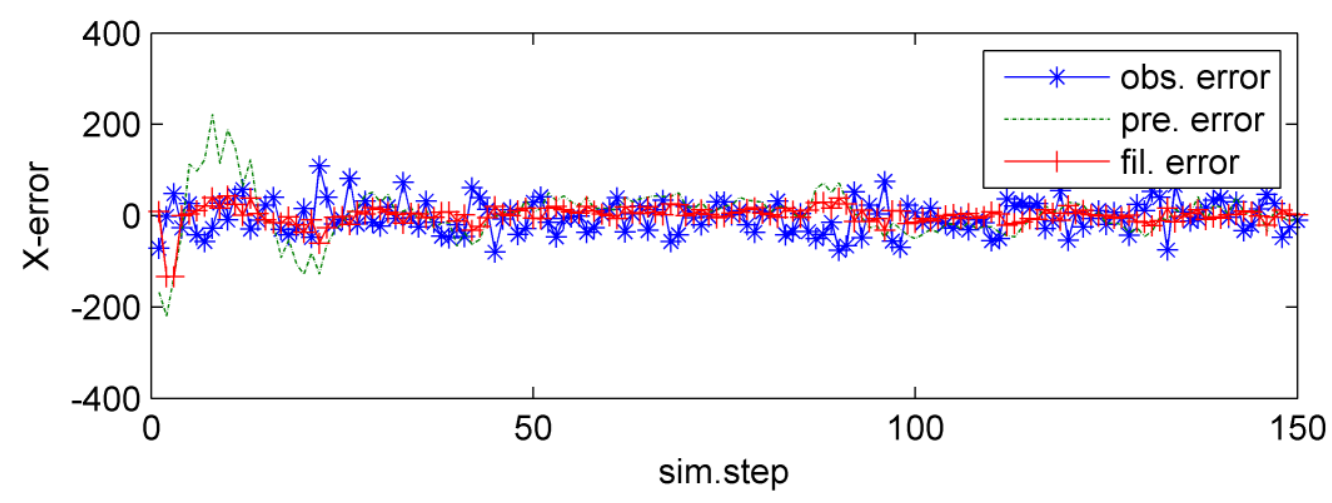

Figure 8. The Error of Observation, Prediction and Filtering In Adaptive Algorithm In X

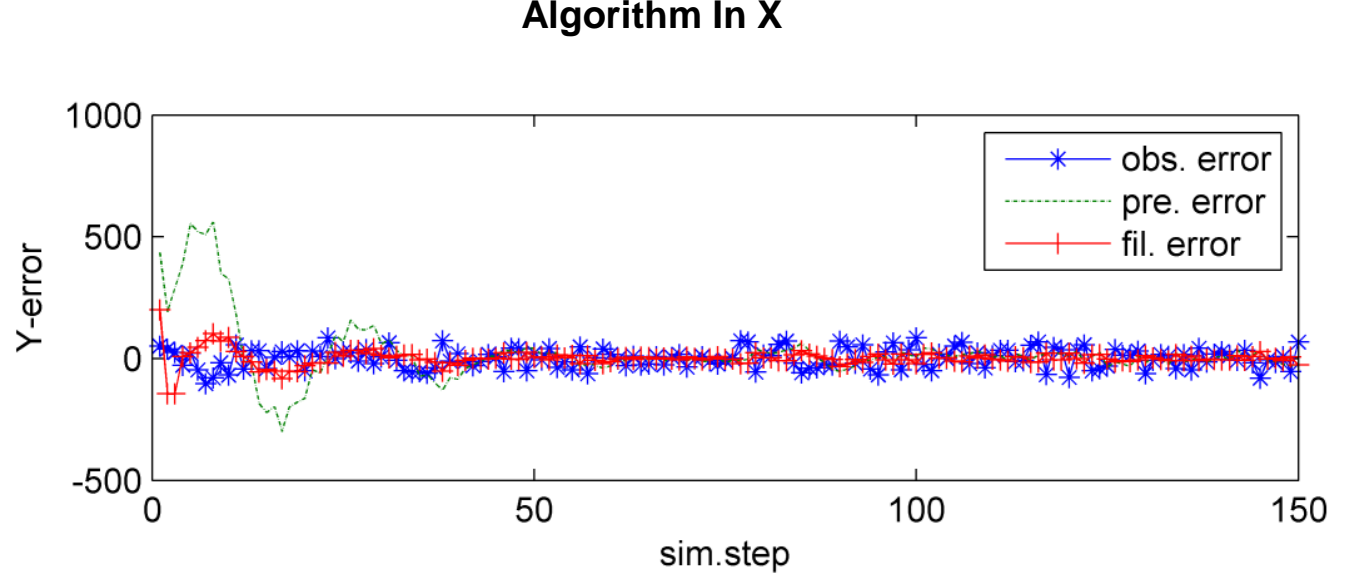

Figure 9. The Error of Observation, Prediction and Filtering In Adaptive Algorithm in $Y$

\section{Conclusion}

Kalman Filtering lechnique has been widely used to solve different filtering problems especially for tracking purposes. Extended Kalman Filtering technique is used for modeling an adaptive Kalman Filtering algorithm for tracking maneuvering trajectory in this paper.

This paper has resolved this apparent problem by demonstrating firstly that standard Kalman Filtering provides tracking performance almost as good as tracking a known nonmaneuvering model, and secondly that is not robust to tracking maneuvering model. The key to this performance degradation is that the model is uncertain and hence too easily lead to great prediction error. An improved Kalman Filtering algorithm has been proposed to improve initial acceleration by using equilibrium optimization solution which tunes the state prediction vector to diminish the error between measured value and prediction estination value. The experimental results have shown that the proposed adaptive algorithm provides better performance than the standard algorithm.

\section{Acknowledgements}

This work was supported by the Education Department of Liaoning Province, General project(No. L2014085), and the Major Technology Platforms of china, Liaoning. 


\section{References}

[1] V. J. Aidala, "Kalman filter behavior in bearings-only tracking applications", Aerospace and Electronic Systems, IEEE Transactions on, vol. 1, (1979), pp. 29-39.

[2] D. Simon, "Kalman filtering", Embedded Systems Programming, vol. 14, no. 6, (2001), pp. 72-79.

[3] A. Amirzadeh, and A. Karimpour, "An interacting Fuzzy-Fading-Memory-based Augmented Kalman Filtering method for maneuvering target tracking”, Digital Signal Processing, vol. 23, no. 5, (2013), pp. 1678-1685.

[4] I. Arasaratnam and S. Haykin, "Cubature kalman filters", Automatic Control, IEEE Transactions on, vol. 54, no. 6, (2009), pp. 1254-1269.

[5] A. Ahmadreza, and M. Hamidreza, "A New Method for Improvement the Accuracy of Augmented Kalman Filter in Presence of High Maneuvers”, International Review of Automatic Control, vol. 5, no. 1, (2012).

[6] Z. Beheshtipour and H. Khaloozadeh, "High Maneuvering Target Tracking Using Fuzzy Covariance Presetting”, Journal of Applied Sciences, vol. 8, no. 20, (2008).

[7] W. Xu, Y. Liu and X. Yin, "Method for underwater target tracking based on an interacting multiple model", Geo-spatial Information Science, vol. 11, no. 3, (2008), pp. 186-190.

[8] W.R. Gilks and C. Berzuini, "Following a moving target-Monte Carlo inference for dynamic Bay esran models", Journal of the Royal Statistical Society: Series B (Statistical Methodology), vol. 63, no. 1, (2001), pp. 127-146.

[9] M. S. Grewal and A. P. Andrews, "Kalman filtering: theory and practice using MATLAB", John Wiley \& Sons, (2011)

[10] B. Akpınar and E. Gülal, "Railway track geometry determination using adaptive Kalman filtering model”, Measurement, vol. 46, no. 1, (2013), pp.639-645

\section{Authors}

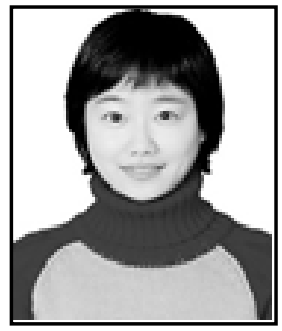

Le Zhang, she received her B.S. degree and M.S. degree in control theory and engineering from Shenyang Ligong University, Shenyang, China, in 2002 and 2005. She is now working toward her Ph.D degree in the Instrument Science and Opto-electronic Engineering Lab at Changehun Unversity of Science and Technology. Her research interests include object tracking, object detection, activity recogntion and opto-electronic intelligent instrument.

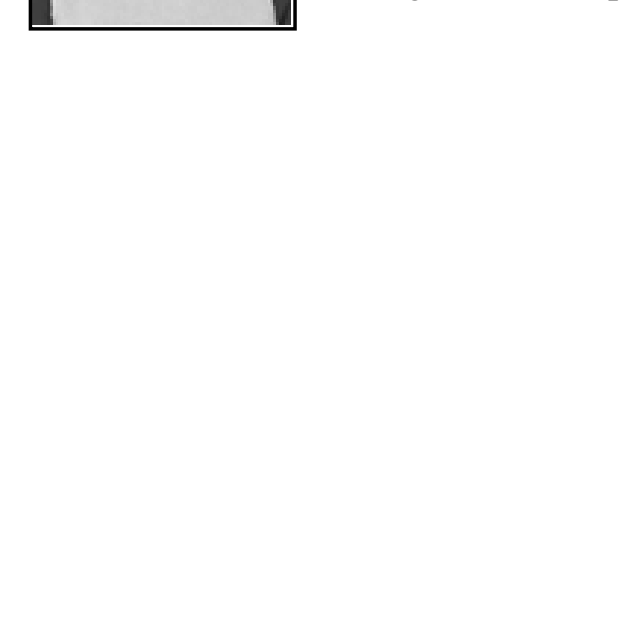


International Journal of Hybrid Information Technology

Vol. 9, No.12 (2016)

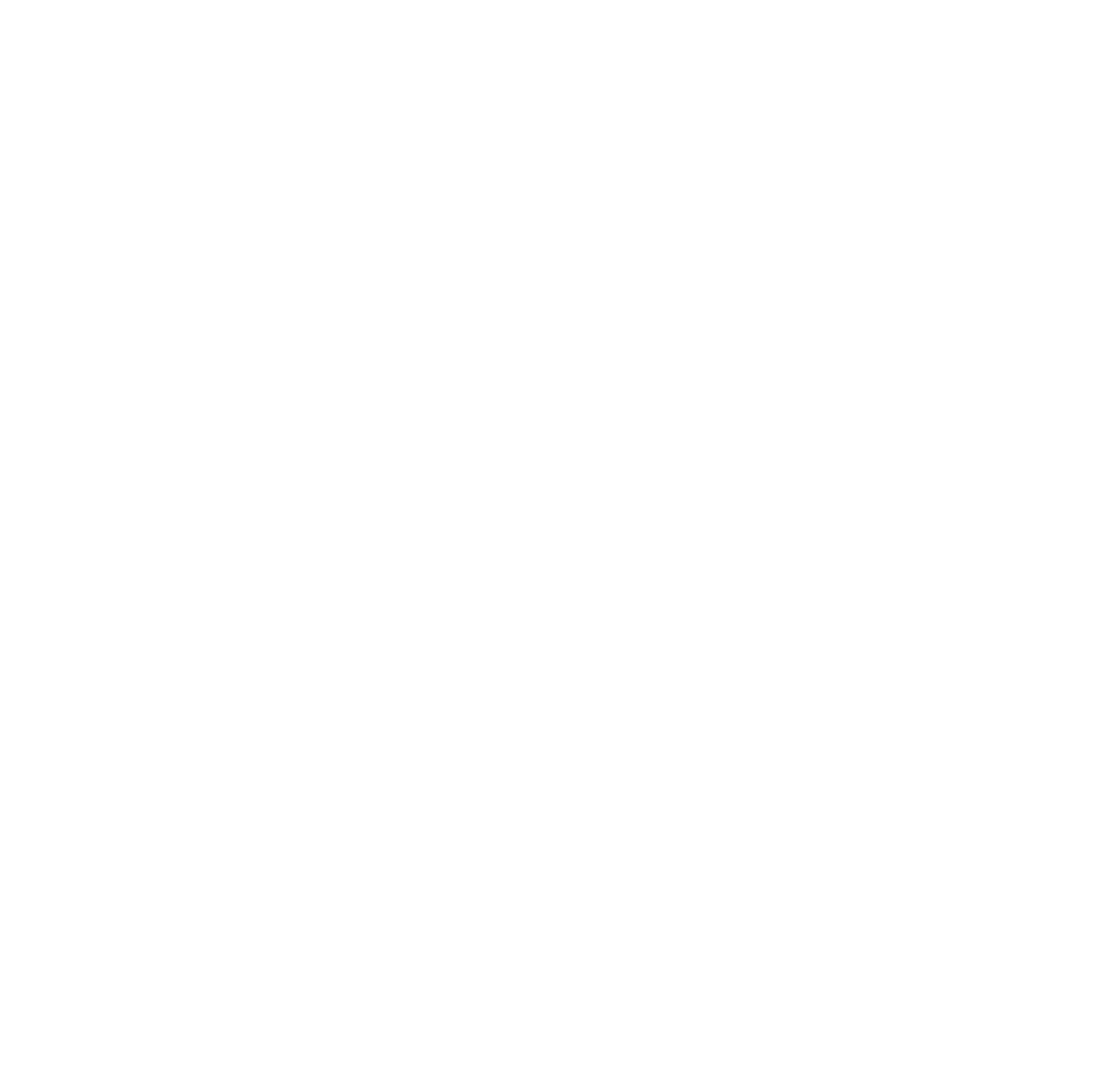

